\title{
HECTD1 regulates the expression of SNAIL: Implications for epithelial-mesenchymal transition
}

\author{
XINGGANG WANG ${ }^{1}$, CHRISTIAN DE GEYTER ${ }^{1,2}$, ZANHUI JIA $^{3}$, YA PENG $^{1}$ and HONG ZHANG ${ }^{1}$ \\ ${ }^{1}$ Department of Biomedicine (DBM); ${ }^{2}$ Reproductive Medicine and Gynecological Endocrinology (RME), \\ University Hospital, University of Basel, CH-4031 Basel, Switzerland; ${ }^{3}$ Second Hospital of Jilin University, \\ Changchun, Jilin 130041, P.R. China
}

Received July 30, 2019; Accepted January 29, 2020

DOI: $10.3892 /$ ijo.2020.5002

\begin{abstract}
As a transcription factor, SNAIL plays a crucial role in embryonic development and cancer progression by mediating epithelial-mesenchymal transition (EMT); however, post-translational modifications, such as ubiquitination, which control the degradation of SNAIL have been observed to affect its functional role in EMT. In a previous study by the authors, it was demonstrated that the HECT domain E3 ubiquitin ligase 1 (HECTD1) regulated the dynamic nature of adhesive structures. In the present study, HECTD1 was observed to interact with SNAIL and regulate its stability through ubiquitination, and the knockdown of HECTD1 increased the expression levels of SNAIL. HECTD1 was discovered to contain putative nuclear localization and export signals that facilitated its translocation between the cytoplasm and nucleus, a process regulated by epidermal growth factor (EGF). Treatment with leptomycin B resulted in the nuclear retention of HECTD1, which was associated with the loss of SNAIL expression. The knockdown of HECTD1 in HeLa cells increased cell migration and induced a mesenchymal phenotype, in addition to
\end{abstract}

Correspondence to: Dr Hong Zhang, Department of Biomedicine (DBM), University Hospital, University of Basel, Hebelstrasse 20, CH-4031 Basel, Switzerland

E-mail: hong.zhang@usb.ch

Abbreviations: EMT, epithelial-mesenchymal transition; CHX, cycloheximide; NLS, putative nuclear localization signal; NES, nuclear export signals; LMB, leptomycin B; EGF, epidermal growth factor; ERK/MAPK, mitogen-activated protein kinase; miR or miRNA, microRNA; E1-E2-E3, RT-qPCR, reverse transcription-quantitative PCR; $\mathrm{CoCl}_{2}$, cobalt chloride; IHC, immunohistochemistry; Halo, haloalkane dehalogenase tag; GFP, green fluorescent protein; XPO1/CRM1, Exportin 1; KD, knockdown; MEF, mouse embryonic fibroblasts cells; Amir, antisense oligonucleotide; COSMIC, the Catalogue Of Somatic Mutations In Cancer; TCGA, The Cancer Genome Atlas

Key words: HECT domain E3 ubiquitin ligase 1, SNAIL, epithelial-mesenchymal transition, nuclear localization, epidermal growth factor receptor signaling, cervical cancer demonstrating sustained EGF signaling, which was observed through increased phosphorylated ERK expression levels. Under hypoxic conditions, HECTD1 expression levels were decreased by microRNA (miRNA or miR)-210. Upon the observation of genetic abnormalities in the HECTDl gene in cervical cancer specimens, it was observed that the decreased expression levels of HECTD1 were significantly associated with a poor patient survival. Thus, it was hypothesized that HECTD1 may regulate EMT through the hypoxia/hypoxia inducible factor $1 \alpha / \mathrm{miR}-210 / \mathrm{HECTD} 1 / \mathrm{SNAIL}$ signaling pathway and the EGF/EGF receptor/HECTD1/ERK/SNAIL signaling pathway in cervical cancer. On the whole, the data of the present study indicated that HECTD1 serves as an E3 ubiquitin ligase to mediate the stability of SNAIL proteins.

\section{Introduction}

Epithelial-mesenchymal transition (EMT) plays an important role in the metastatic process of cancer by facilitating cell migration and invasion. One of the hallmarks of EMT is to acquire mesenchymal traits through the regulation of core EMT-inducing transcriptional factors, such as increasing SNAIL/SLUG expression levels and decreasing the expression levels of E-cadherin. Several E3-ubiquitin ligases have been reported to play crucial roles in the regulation of EMT (1-3), and genetic aberrations and alterations in these ligases have been detected in numerous types of cancer (4-6).

Ubiquitination is a molecular process in which a ubiquitin protein is attached to a substrate protein; it is mediated by E1/E2/E3 multi-enzyme cascades, whereby E1 enzymes are known as the ubiquitin-activating enzymes, E2 enzymes are referred to as the ubiquitin-conjugating enzymes and E3 enzymes are the ubiquitin-protein ligases (7). Ubiquitination is one of the most important enzymatic post-translational modifications to occur that can regulate the function of proteins; it can mark proteins for degradation via the proteasome and it can alter both the cellular location and activity of proteins (8-10).

In a previous study, it was demonstrated that HECT domain E3 ubiquitin ligase 1 (Hectdl) homozygous mutant embryos exhibited numerous defects in embryonic and fetal development (11) and MEF cells isolated from Hecdtl-mutant mice were observed to accelerate cell migration (12). Consistent with these observations, the present study demonstrated that 
the migration of human cells in which HECTD1 was knocked down (HECTD1-KD cells) was altered, which simultaneously suppressed the degradation of SNAIL. SNAIL is a transcriptional factor. It exerts global effects on gene expression and it promotes EMT during in embryonic development and cancer progression, we then hypothesized that HECTD1 plays a role in EMT via regulating SNAIL expression. Thus, the present study aimed to investigate the role that HECTD1 plays in regulating the expression of SNAIL and to elucidate the association between the HECTD1-mediated degradation of SNAIL and the EMT pathway.

\section{Materials and methods}

Cell culture and reagents. HeLa cells were obtained from the American Type Culture Collection (CCL-2) and Ca Ski cells (87020501) were purchased from the European Collection of Authenticated Cell Cultures. HeLa cells were cultured in DMEM, supplemented with 10\% FBS (Sigma-Aldrich; Merck KGaA), 1,000 mg/l glucose (Sigma-Aldrich; Merck KGaA), $2 \mathrm{mM}$ L-Glutamine (Sigma-Aldrich; Merck KGaA) and $1 \mathrm{mM}$ sodium pyruvate (Sigma-Aldrich; Merck KGaA). Ca Ski cells were cultured in RPMI-1640 medium (Sigma-Aldrich; Merck $\mathrm{KGaA}$ ), supplemented with $10 \% \mathrm{FBS}$ and $2 \mathrm{mM}$ L-Glutamine. All cells were maintained in a humidified atmosphere of $5 \% \mathrm{CO}_{2}$ and $37^{\circ} \mathrm{C}$.

To mimic hypoxic conditions, $1 \times 10^{5}$ cells were treated with $500 \mu \mathrm{M}$ cobalt chloride $\left(\mathrm{CoCl}_{2}\right)$ for $0-60$ min or 8,16 and $24 \mathrm{~h}$. For EGF treatment, $5 \times 10^{4} \mathrm{HeLa}$ cells were seeded into 24-well plates containing 12-mm round glass cover slips or on $100-\mathrm{mm}$ dishes. Following $24 \mathrm{~h}$ of incubation at $37^{\circ} \mathrm{C}$, the cells were serum-starved overnight and subsequently treated with $100 \mathrm{ng} / \mathrm{ml}$ EGF for various times.

Cell transfection. Different clones of the HECTD1 KD in HeLa or Ca Ski cells were established through the stable transfection of individual of 4 short hairpin (sh)RNAs (cat. no. TF304134; OriGene Technologies, Inc., which consists of 4 shRNAs against HECTD1, cat. nos. FI316529, FI316530, FI316531 and F1316532). The control cells (Ctrl, NC) were stably transfected with an empty shRNA RFP Cloning Vector (scramble, pRFP-C-RS vector; cat. no. TR30014; OriGene Technologies, Inc.). The RFP expression cassettes in the shRNA constructs were excised with $X h o \mathrm{I} / B g l \mathrm{II}$ and blunt-end ligation.

miRTarBase was used to identify hypothetical target sequences for HECTD1 (http://mirtarbase.mbc.nctu.edu. tw/php/search.php). microRNA (miR)-21 and miR-210 antagomirs were purchased from Ambion; Thermo Fisher Scientific, Inc. and the scrambled negative control (anti-miR-scr) was obtained from Shanghai GenePharma Co. Ltd. These antagomirs have been previously described and verified (13-15). Cells were transfected with $50 \mathrm{nM} \mathrm{miR-21/-210}$ antagomir or $25 \mathrm{nM}$ anti-miR-scr using RNAifectin ${ }^{\mathrm{TM}}$ (ABM, Inc.), according to the manufacturer's protocol.

Reverse transcription-quantitative PCR (RT-qPCR). $h s a-m i R-21-5 p$ and $h s a-m i R-210-3$ RT-qPCR kits (Applied Biosystems; Thermo Fisher Scientific, Inc.) were used for RT-qPCR. RT-qPCR was performed using a TaqMan PCR kit (Applied Biosystems; Thermo Fisher Scientific, Inc.), according to the manufacturer's protocol. The following primers were used for qPCR: U6 forward, 5'-CTCGCTTCGGCAGCA CA-3' and reverse, 5'-AACGCTTCACGAATTTGCGT-3'; and HECTD1 forward, 5'-AATGAACCAGGGTCAACT GC-3' and reverse, 5'-TGTGTTTGTCCACTGGCATT-3'. The cycling conditions were as follows: $95^{\circ} \mathrm{C}$ for $10 \mathrm{~min}$, followed by 30 cycles of $\left(95^{\circ} \mathrm{C}\right.$ for $15 \mathrm{sec}, 60^{\circ} \mathrm{C}$ for $30 \mathrm{sec}, 72^{\circ} \mathrm{C}$ for $40 \mathrm{sec}$ ), then $95^{\circ} \mathrm{C}$ for $60 \mathrm{sec}$. Expression levels were calculated using the $2^{-\Delta \Delta \mathrm{Cq}}$ method (16) and normalized to the loading control U6. All experiments were performed $>3$ times and expressed as a $\log _{2}$ scale or fold change of treatment/control. Expression changes with a $\log _{2}$ ratio of treatment/control $>1$ or $<-1$ and $\mathrm{P}<0.05$ were considered significant.

Antibodies and reagents. Anti-SNAIL (cat. no. 3879), anti-SLUG (cat. no. 9585), anti-E-cadherin (cat. no. 3195), anti-N-cadherin (cat. no. 4061), anti-ERK1/2 (cat. no. 4695), anti-phospho-ERK1/2 (Thr202/Tyr204; cat. no. 4374) and anti-GAPDH (2118L) primary antibodies were purchased from Cell Signaling Technology, Inc.; the anti-HECTD1 primary antibody (cat. no. CSB-PA010273GA01HU) was obtained from Cusabio Technology LLC; the anti-ubiquitin primary antibody (cat. no. ab7780) was obtained from Abcam; and the anti-HECTD1 (M03), clone 1E10 primary antibody (cat. no. H00025831-M03) was purchased from Abnova Corp. The FITC-conjugated and Alexa Fluor 546-conjugated anti-rabbit (A-11035) or anti-mouse (A-11003) secondary antibodies were purchased from Invitrogen; Thermo Fisher Scientific, Inc. and the horseradish peroxidase (HRP)-conjugated anti-mouse (cat. no. 1706516) or anti-rabbit (cat. no. 1706515) secondary antibodies were obtained from Bio-Rad Laboratories, Inc. Anti- $\alpha$-tubulin antibody was obtained from Abcam (ab52866, 1:250). Human recombinant EGF and EGF-Alexa Fluor 488 were purchased from Invitrogen; Thermo Fisher Scientific, Inc. The GFP-SNAIL plasmid (cat. no. 16225) was obtained from Addgene, Inc. and the HECTD1-HaloTag ${ }^{\circledR}$ human ORF in pN21A clone (cat. no. FHC05410) was purchased from Promega Corp. Leptomycin B (LMB; cat. no. L2913) and ivermectin (IVE; cat. no. I8898) were purchased from Sigma-Aldrich; Merck $\mathrm{KGaA}$.

Cycloheximide (CHX) chase assay and LMB or IVE treatments. Briefly, $2.5 \times 10^{6}$ shRNA-transfected HeLa cells were plated on $100-\mathrm{mm}$ dishes for $16 \mathrm{~h}$. Following the treatment with $100 \mu \mathrm{g} / \mathrm{ml} \mathrm{CHX}$ for the indicated time periods, cell pellets were harvested and lysed on ice in the supplemented RIPA buffer. Protein expression was detected by western blot analysis and quantified by densitometric analysis with ImageJ software. Three independent experiments were performed. For LMB or IVE treatments, HeLa cells were treated with or without $50 \mathrm{nM}$ LMB or IVE $(1 \mu \mathrm{M})$ for $4 \mathrm{~h}$ and sequentially treated with $100 \mathrm{ng} / \mathrm{ml} \mathrm{EGF}$.

Immunofluorescence staining. Cells were seeded into 24-well plates containing $12-\mathrm{mm}$ round cover glass slips. Prior to treatment, the cells were serum-starved for $16 \mathrm{~h}$. Following treatment, the cells were washed twice with PBS and fixed with $4 \%$ formaldehyde for $15 \mathrm{~min}$ at room temperature. The cells 
were subsequently permeabilized with $0.15 \%$ Triton-X100/PBS for $15 \mathrm{~min}$ at room temperature and washed twice with PBS. The cells were then blocked with 5\% BSA/PBS for $1 \mathrm{~h}$ at room temperature and incubated at $4^{\circ} \mathrm{C}$ overnight with anti-HECTD1 and anti-SNAIL primary antibodies diluted 1:200 in blocking buffer. Following primary antibody incubation, the slides were washed with PBS 3 times for $15 \mathrm{~min}$ and were subsequently incubated with Alexa Fluor-conjugated secondary antibodies (1:500) for $1 \mathrm{~h}$ at room temperature. The slides were then washed 3 times with PBS and the nuclei were stained with DAPI for $3 \mathrm{~min}$ at room temperature. The cells were subsequently mounted with prolonged gold anti-fade mountant (Invitrogen; Thermo Fisher Scientific, Inc.) and the stained cells were visualized using the Nikon A1R confocal microscope (Nikon Corp.).

The fluorescent intensities of HECTD1 and SNAIL were quantified using NIS-Elements software (Nikon Corp.). The nuclear regions were selected with the DAPI channel and the measured intensities were subtracted from the background intensity (non-cell region) to obtain the fluorescent intensity of each protein. At least 3 independent experimental repeats were performed.

Analysis of HECTD1 subcellular localization. The analysis of the subcellular localization of HECTD1 was performed using two different prediction softwares: RSLpred (17) and PSORT II (18).

Western blot analysis. The cells were washed twice with pre-chilled PBS at $4^{\circ} \mathrm{C}$ and total protein was extracted on ice for 10 min using $200 \mu \mathrm{l}$ RIPA lysis buffer, containing $10 \%$ proteinase inhibitor. The cells were collected into a $1.5 \mathrm{ml}$ Eppendorf tube, vortexed and lysed on ice for $30 \mathrm{~min}$. Subsequently, the cells were centrifuged for $15 \mathrm{~min}$ at $6,500 \mathrm{x}$ g at $4^{\circ} \mathrm{C}$ and the supernatant was collected. Total protein was quantified using a protein assay (Bio-Rad Laboratories, Inc.) and $20 \mu \mathrm{g}$ protein/lane were separated on 12\% SDS-PAGE to detect ERK1/2 and phospho-ERK1/2 expression and 4\% SDS-PAGE to detect HECTD1 expression. The separated proteins were subsequently transferred onto PVDF membranes and blocked with $1 \%$ milk powder. The membranes were incubated with the following primary antibodies at $4^{\circ} \mathrm{C}$ overnight: Anti-ERK1/2 (1:200), anti-phospho-ERK1/2 (1:1,000), anti-HECTD1 (1:200) and anti-GAPDH (1:100,000). Following primary antibody incubation, the membranes were incubated with HRP-conjugated secondary antibodies $(1: 5,000)$ for $1 \mathrm{~h}$ at room temperature. Protein bands were visualized using the Pierce $^{\mathrm{TM}}$ ECL Plus Substrate (Pierce; Thermo Fisher Scientific, Inc.). Protein expression was quantified using ImageJ software (National Institutes of Health) and normalized to the loading control, GAPDH.

Cell proliferation assays. To analyze cell proliferation, the CellTiter $96^{\circledR} \mathrm{AQ}_{\text {ueous }}$ One Solution Cell Proliferation assay (MTS; cat. no. G3582; Promega Corp.) was used according to the manufacturer's protocol. Following serum starvation overnight, a total of $5 \times 10^{3}$ cells/well were seeded into 96-well plates with or without $100 \mathrm{ng} / \mathrm{ml}$ EGF treatment. Following incubation for 30,48 and $72 \mathrm{~h}, 20 \mu \mathrm{l}$ MTS solution were added to each well and the cells were incubated for $3 \mathrm{~h}$ at $37^{\circ} \mathrm{C}$. The absorbance was measured at $490 \mathrm{~nm}$ using a Biotek 96-well plate reader (BioTek Instruments, Inc.). The absorbance was normalized to the absorbance at $0 \mathrm{~h}$. A single experiment was performed.

Wound healing assay. The wound healing assay for HeLa cells was performed as previously described (12). Three independent experimental repeats were performed.

Cell migration assay. A total of $4 \times 10^{5} \mathrm{Ca}$ Ski or HeLa cells $/ \mathrm{ml}$ were resuspended in serum-free DMEM and $350 \mu \mathrm{l}$ cell suspension was plated in the upper chambers of 24-well Transwell plates $(8.0 \mu \mathrm{m}$; cat. no. MCEP24H48; Merck KGaA). A total of $1 \mathrm{ml}$ DMEM or RPMI-1640 medium supplemented with $20 \%$ FBS was plated in the lower chambers. Following incubation for $20 \mathrm{~h}$ at $37^{\circ} \mathrm{C}$, the non-migratory cells remaining in the upper chamber were removed using cotton swabs. The migratory cells were fixed with $70 \%$ ethanol for $15 \mathrm{~min}$ and subsequently stained with $0.1 \%$ crystal violet for $15 \mathrm{~min}$ at room temperature. The stained cells were counted in four randomly selected fields using a microscope (ECLIPSE Ti2, Nikon). Three independent experimental repeats were performed.

Co-immunoprecipitation (Co-IP). Cell pellets were lysed with IP lysis buffer (20 mM Tris-HCl, pH 8.0, $137 \mathrm{mM}$ $\mathrm{NaCl}, 1 \% \mathrm{NP} 40$ and $2 \mathrm{mM}$ EDTA supplemented with $1 \%$ protease inhibitor cocktail) on ice for $20 \mathrm{~min}$. The lysate was centrifuged at $9,000 \mathrm{xg}$ for $5 \mathrm{~min}$ at $4^{\circ} \mathrm{C}$ and the supernatant was collected and transferred to pre-cooled fresh tubes. The protein concentration was equilibrated with the IP lysis buffer. Subsequently, $2 \mu \mathrm{l}$ anti-GFP GF28R antibody (Thermo Fisher Scientific, Inc.) were added per $500 \mu \mathrm{g}$ protein sample prior to being incubated overnight at $4^{\circ} \mathrm{C}$. Normal mouse IgG (1:1,000, ab188776, Abcam) was used as a negative control. The lysates were subsequently incubated with pre-washed protein $\mathrm{G}$ agarose beads $\left(20 \mu \mathrm{l} / 500 \mu \mathrm{g}\right.$ protein) for $1 \mathrm{~h}$ at $4^{\circ} \mathrm{C}$ with gentle agitation. The beads were washed 3 times with IP lysis buffer and then centrifuged at $1,600 \mathrm{xg}$ for $3 \mathrm{~min}$ at $4^{\circ} \mathrm{C}$. Subsequently, the beads were heated for $5 \mathrm{~min}$ at $95^{\circ} \mathrm{C}$ in $2 \mathrm{X}$ Laemmli sample buffer and target proteins were detected by western blot analysis using specific antibodies as described above. Two independent experimental repeats were performed.

Ubiquitination assay. Following $24 \mathrm{~h}$ of transfection with GFP-SNAIL or GFP, HeLa cells were washed twice with PBS and incubated in serum-free medium supplemented with $1 \mathrm{nM}$ MG132 or DMSO overnight at $37^{\circ} \mathrm{C}$. For the endogenous ubiquitination assay, starved cells were harvested as pellets and resuspended in serum-free medium. The pellets were centrifuged $9,000 \mathrm{x}$ g for $5 \mathrm{~min}$ at $4^{\circ} \mathrm{C}$ and lysed with ubiquitination lysis buffer (50 mM Tris, pH 7.5, 1 mM EDTA, $150 \mathrm{mM}$ $\mathrm{NaCl}, 0.1 \%$ Triton X-100, complete protease inhibitor cocktail, $100 \mu \mathrm{M}$ MG132 and $100 \mu \mathrm{M}$ N-ethylmaleimide) on ice for $15 \mathrm{~min}$, followed by centrifugation at $12,000 \mathrm{x}$ g for $5 \mathrm{~min}$ at $4^{\circ} \mathrm{C}$. The supernatant was collected and used to determine the total protein concentration. Equal concentrations of total protein were immunoprecipitated with GFP antibody and protein $\mathrm{G}$ agarose beads, and ubiquitination was subsequently detected using western blotting. Whole cell lysate, which was $5 \%$ of total protein, was used as the control. Two independent experimental repeats were performed. 
Clinical specimens and immunohistochemistry. The expression levels of HECTD1 were examined in normal and cancer tissues obtained from the Human Protein Atlas (HPA; http://www.proteinatlas.org) using immunohistochemistry (IHC). Briefly, IHC was performed using anti-HECTD1 (1:50, cat. no. HPA002929; Sigma-Aldrich; Merck KGaA) and anti-SNAIL (1:500, cat no. HPA069985; Sigma-Aldrich; Merck KGaA) primary antibodies, and the source of these tumors and the pathological analysis can be found in the online supplementary materials. Kaplan-Meier plots were generated based on the association analysis between mRNA expression levels and patient survival, and patients were subsequently divided into 2 groups based on the level of expression as the 'Low' or 'High' expression groups. Log-rank P-values are displayed. 329 cervical cancer samples from The Catalogue Of Somatic Mutations In Cancer (COSMIC) and 309 samples from The Cancer Genome Atlas (TGCA) were analyzed using whole-exome sequencing.

Statistical analysis. Statistical analysis was performed using unpaired one-way ANOVA (Tukey's multiple comparisons test was used as a post-hoc test) or subsequent Student's t-test using GraphPad Prism 7 software (GraphPad Software, Inc.) and data are presented as the means \pm SD or SEM, as indicated in the figure legends. $\mathrm{P}<0.05$ was considered to indicate a statistically significant difference.

\section{Results}

Interaction of HECTDI with SNAIL. It has previously been demonstrated that the degradation of SNAIL occurs through a proteasome-mediated mechanism involving several E3 ubiquitin ligases (19-21). SNAIL has been suggested to interact with HECTD1, a HECT domain E3 ubiquitin ligase involved in regulating the dynamic nature of adhesive structures in cells (11). Therefore, it was hypothesized that HECTD1 may be involved in the protein degradation of SNAIL. In this study, Co-IP assays were used to investigate whether HECTD1 physically interacted with SNAIL protein; HECTD1 was present in the immunoprecipitates of cell lysates transfected with an expression vector for GFP-SNAIL, but not in those collected following transfection with the control vector alone (Fig. 1A), although endogenous SNAIL could not be detected in a pulldown in HaloTag-HECTD1 transfected cells. These results suggest that the proteins interact with each other in vivo. To elucidate the potential role of HECTD1 in cancer, several cell lines were established in which HECTD1 was knocked down (HECTD1-KD cells) using shRNA. One of these HECTD1-KD cells established from TF304134, demonstrated markedly reduced expression levels compared with the scrambled shRNA-transfected cells (Ctrl); $>90 \%$ of HECTD1 expression was knocked down in HeLa cells (Fig. S1A). Since HECTD1 is an E3 ubiquitin ligase, the HECTD1-mediated ubiquitination of SNAIL was investigated. The GFP-SNAIL vector was overexpressed in HeLa cells and ubiquitinated proteins were purified using anti-GFP antibody beads, and SNAIL expression was subsequently analyzed by western blot analysis. The ubiquitination of SNAIL was markedly increased following treatment with the proteasome inhibitor, MG132, which blocks ubiquitin-proteasome degradation (Fig. 1B). Furthermore, the HECTD1-KD cells were observed to have reduced ubiquitination levels of SNAIL (Fig. 1B), whereas cells transfected with expression pN21A plasmids containing HECTD1 (HaloTag ${ }^{\circledR}$-HECTD1) and the GFP-SNAIL plasmid demonstrated that HECTD1 expression promoted the ubiquitination of SNAIL (Fig. 1C). Overall, these data suggest that SNAIL proteins are the target of HECTD1-mediated ubiquitination.

Mediation of SNAIL degradation by HECTD1. To identify whether HECTD1 is involved in the degradation of SNAIL, the CHX chase assay was used. Compared with the control cells (Ctrl), cells transfected with HECTD1-KD exhibited markedly decreased degradation levels of SNAIL proteins (Figs. 2A and S2), suggesting that HECTD1 may be one of the E3 ubiquitin ligases that mediates the stability of SNAIL proteins.

It was subsequently hypothesized that the localization of HECTD1 within cells may affect HECTD1-mediated SNAIL degradation. Thus, to examine this hypothesis, the localization of SNAIL in HeLa Ctrl and HECTD1-KD cells was analyzed. Consistent with the results obtained by western blot analysis (Fig. 2A), it was observed that the immunoreactivity of nuclear SNAIL proteins was increased in the HECTD1-KD cells compared with the Ctrl cells (Fig. 2B and C). In contrast to the localization of HECTD1 (Fig. 3A), SNAIL expression was found to be constitutive and dependent on serum/growth factors in HeLa cells (Fig. 2B). Compared with serum starvation (-EGF), the immunoreactivity of nuclear SNAIL protein was increased following the addition of EGF (Fig. 2B and D); however, SNAIL was predominantly expressed in the nucleus of the HECTD1-KD cells, regardless of the treatment condition. These results strongly suggest that HECTD1 may play an important role in controlling the abundance of SNAIL present in the nucleus.

Shuttling of HECTD1 between the nucleus and cytoplasm requires Exportin 1 (XPO1/CRM1)-mediated nuclear export. HECTD1 contains 8 putative nuclear localization sequences (NLS) and 4 nuclear export signals (NES; Fig. S3A and B). Through using two localization prediction software, it was discovered that HECTD1 expression was localized within the nucleus (Fig. S3C). Consistent with this finding, endogenous HECTD1 was also found to be localized in the nucleus following serum deprivation, despite its otherwise cytoplasmic localization (Fig. 3A and B), which is similar to FBXL5 (19), but unlike many other types of SNAIL1 E3 ubiquitin ligases, which are localized in the cytosol (21). Notably, EGF treatment stimulated the export of HECTD1 to the cytosol (Fig. 3C and D), indicating that HECTD1 may shuttle back and forth between nucleus and cytoplasm. No changes were observed in the intensity of HECTD1 immunoreactivity during the 4-h period (Fig. 3A and B).

It has been demonstrated that XPO1/CRM1 mediates the nuclear export of numerous types of protein (22). LMB, a well-known natural inhibitor of XPO1/CRM1, has been observed to reduce nuclear export (23). The treatment of HeLa cells with LMB resulted in the accumulation of HECTD1 in the nucleus (Fig. 3C and D), suggesting that HECTD1 may be actively exported from the nucleus to the cytosol.

To investigate the location of HECTD1-mediated SNAIL degradation by ubiquitination, the nuclear export or import of HECTD1 was blocked by LMB or IVE (which is a specific inhibitor of importin $\alpha / \beta$-mediated nuclear import), 


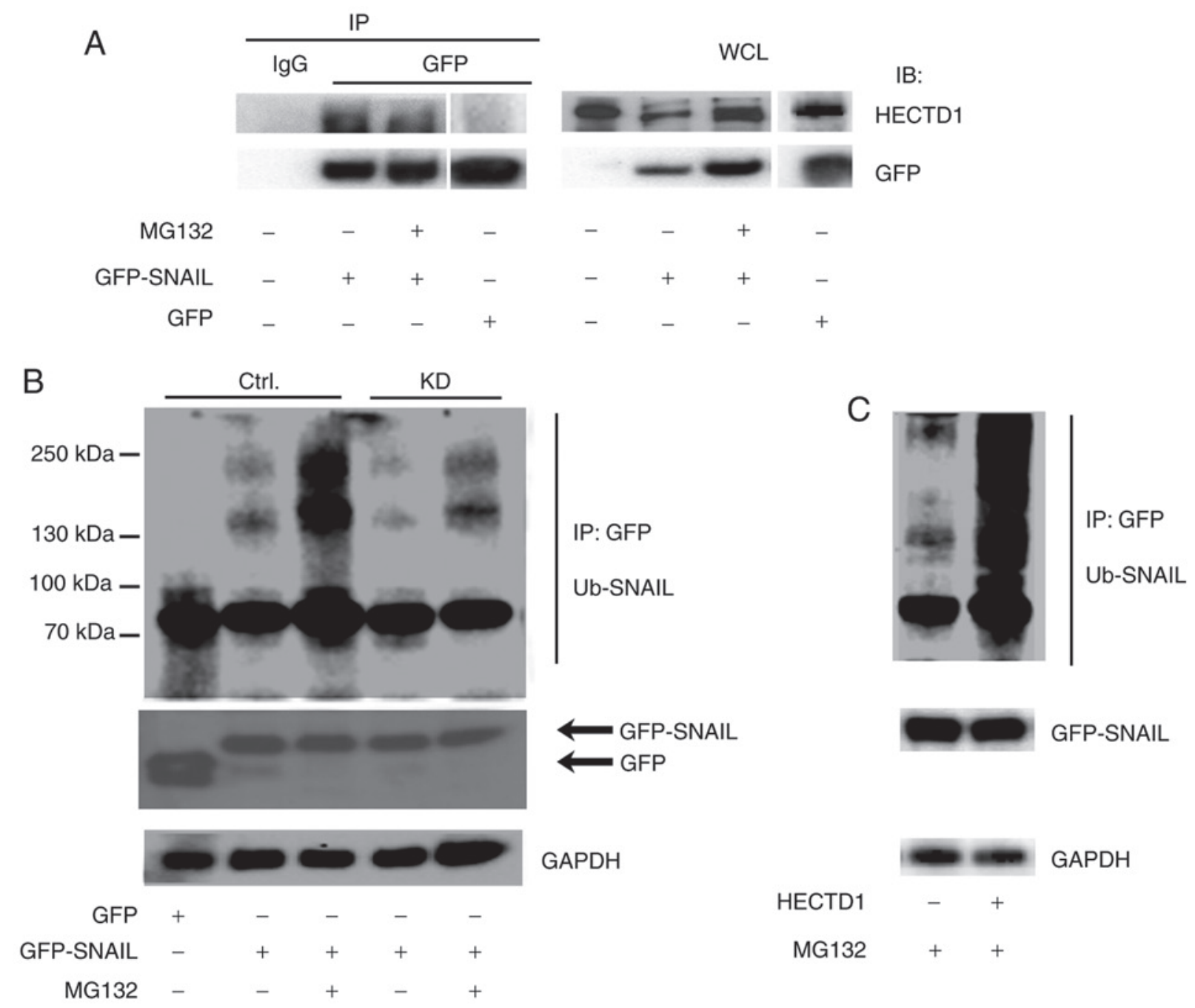

Figure 1. Interaction of HECTD1 with SNAIL. (A) In vivo interaction of HECTD1 with SNAIL. The interaction between HECTD1 and SNAIL expression levels were investigated using Co-Immunoprecipitation. HeLa cells were transfected with GFP-tagged SNAIL or GFP for $24 \mathrm{~h}$, followed by sequential treatment with DMSO or $5 \mu \mathrm{M}$ MG132 for $16 \mathrm{~h}$. The cell lysates were immunoprecipitated with anti-GFP. IPs and WCLs were analyzed using western blotting to detect the expression levels of HECTD1 and GFP. (B) SNAIL ubiquitination assay. HeLa Ctrl and HECTD1-KD cells were transiently transfected with GFP-SNAIL or GFP empty vector for $24 \mathrm{~h}$, followed by sequential treatment with DMSO or $5 \mu \mathrm{M}$ MG132 for $16 \mathrm{~h}$. The cell lysates were immunoprecipitated with anti-GFP antibody. IPs and WCLs were analyzed by western blot analysis with anti-ubiquitin, anti-GFP and anti-GAPDH antibodies. Results are representative of 2 experimental repeats. (C) HECTD1 promotes the ubiquitination of SNAIL in vivo. HeLa cells were transfected with expression plasmids for HECTD1 (Halo-HECTD1) and SNAIL (GFP-SNAIL) in the presence of $5 \mu \mathrm{M}$ MG132 for $16 \mathrm{~h}$. The cell lysates were immunoprecipitated with anti-GFP antibody and analyzed by western blot analysis with anti-ubiquitin antibodies. IP, immunoprecipitates; WCL, whole cell lysates; Ctrl, negative control; KD, knockdown; HECTD1, HECT domain E3 ubiquitin ligase 1.

respectively, and the nuclear signals and expression levels of SNAIL were determined by fluorescence intensity. LMB not only increased the nuclear signal of HECTD1, but also that of SNAIL (Fig. 3E and F); however, it was suggested that the nuclear localization of SNAIL was independent of XPO1/CRM1 in HeLa cells. The total expression levels of SNAIL were unaltered (Fig. 3G), indicating that LMB may block the nuclear export of both proteins, but the degradation of SNAIL does not occur in the nucleus. IVE treatment blocked the nuclear import of both proteins (Fig. 3E and F) and decreased the total SNAIL expression levels by $30 \%$ (Fig. 3G). Overall, these results suggest that HECTD1-mediated SNAIL degradation occurs in the cytoplasm, but not in the nucleus.

Knockdown of HECTDl potentiates EMT. Alterations in cellular adhesion, migration, invasion and morphology are essential for EMT. The knockdown of HECTD1 induced a mesenchymal appearance in the HeLa cells (Fig. 4A). The Ctrl HeLa cells displayed a 'cobblestone (cubed)', epithelial-like phenotype (24), whereas the HECTD1-KD cells acquired a spindle-like, elongated phenotype, which is typical of mesenchymal cells, suggesting that the knockdown of HECTD1 may induce changes in cell morphology similar to EMT processes.

MEF cells obtained from Hectdl-homozygous mutant $\left(\right.$ Hectdl $\left.1^{R / R}\right)$ embryos exhibited an accelerated cell spreading/migratory phenotype (11). Similarly, the wound healing assays demonstrated that closure of the wound occurred more rapidly in the HECTD1-KD compared with the Ctrl cells (Fig. 4B); furthermore, in the Transwell assay, the number of migrated cells was higher in KD cells as compared to the Ctrl cells (Fig. 4C). However, the knockdown of HECTD1 in the HeLa cells did not significantly modify the proliferation rate 

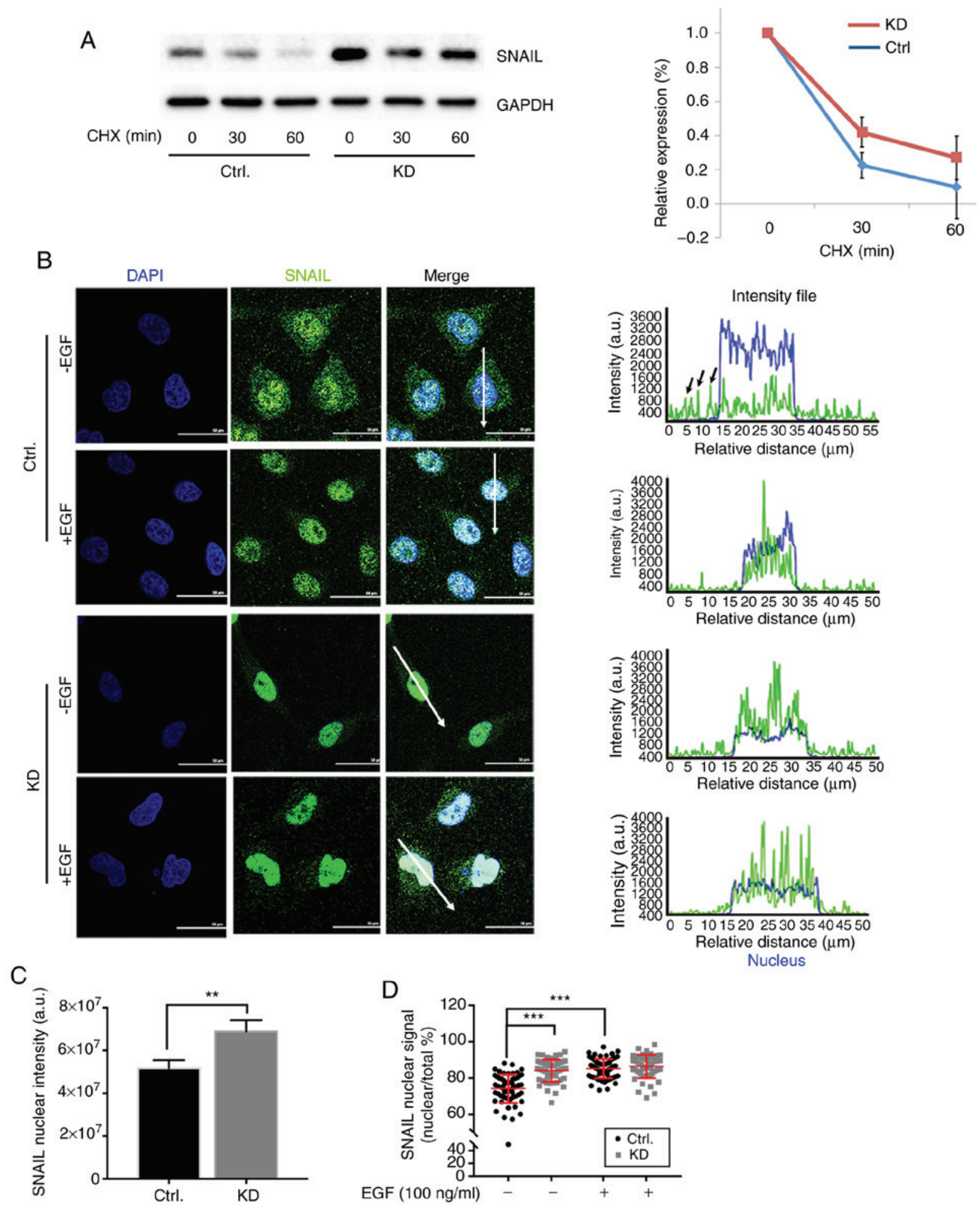

Figure 2. Subcellular localization of SNAIL. (A) CHX chase assay. HeLa cells were treated with $100 \mu \mathrm{g} / \mathrm{ml} \mathrm{CHX}$ for the indicated time period and western blot analysis was performed with an anti-SNAIL antibody. Statistical analysis was performed using the Student's t-test. (B) Subcellular localization of SNAIL was analyzed using fluorescence microscopy in the Ctrl- or HECTD1-KD-transfected cells. The subcellular localization of SNAIL in individual cells is indicated with the arrow-line. Scale bar, $50 \mu \mathrm{m}$. (C) SNAIL nuclear signal intensities in Ctrl- and HECTD1-KD cells was examined by staining using anti-SNAIL antibodies. Data are represented as the means \pm SD. ${ }^{* *} \mathrm{P}<0.01$ and $>50$ cells of each cell type was measured. (D) SNAIL nuclear signal in Ctrl- and HECTD1-KD cells with/without epidermal growth factor treatment was analyzed and the percentage of cells exhibiting high levels of nuclear signals are presented. Data are analyzed by one-way ANOVA and represented as the means \pm SD. ${ }^{* * *} \mathrm{P}<0.001$. Ctrl, negative control; HECTD1, HECT domain E3 ubiquitin ligase 1; KD, knockdown; CHX, cycloheximide.

of the cells (Fig. S1B). Thus, the downregulation of HECTD1 expression levels may be strongly associated with abnormal cell migration and invasion, but not with proliferation.
In addition, the alteration in the expression levels of specific transcription factors, such as SNAIL and SLUG, are also typical features of EMT (20). It has been reported 
A
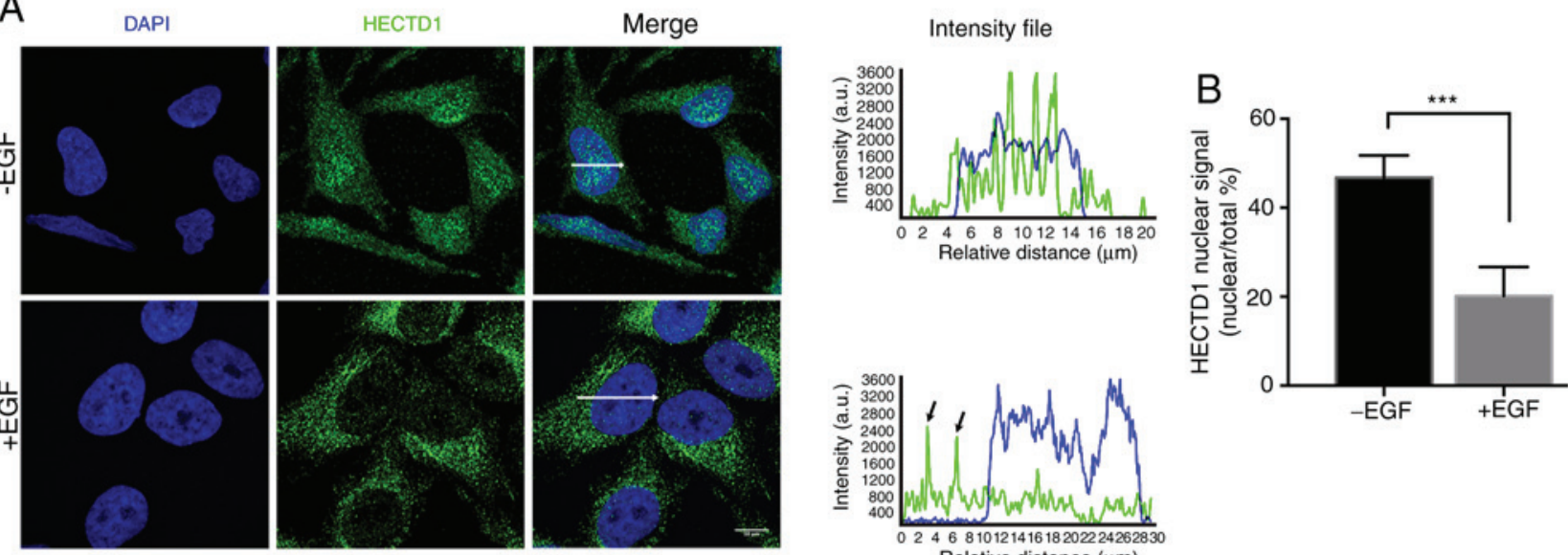

C
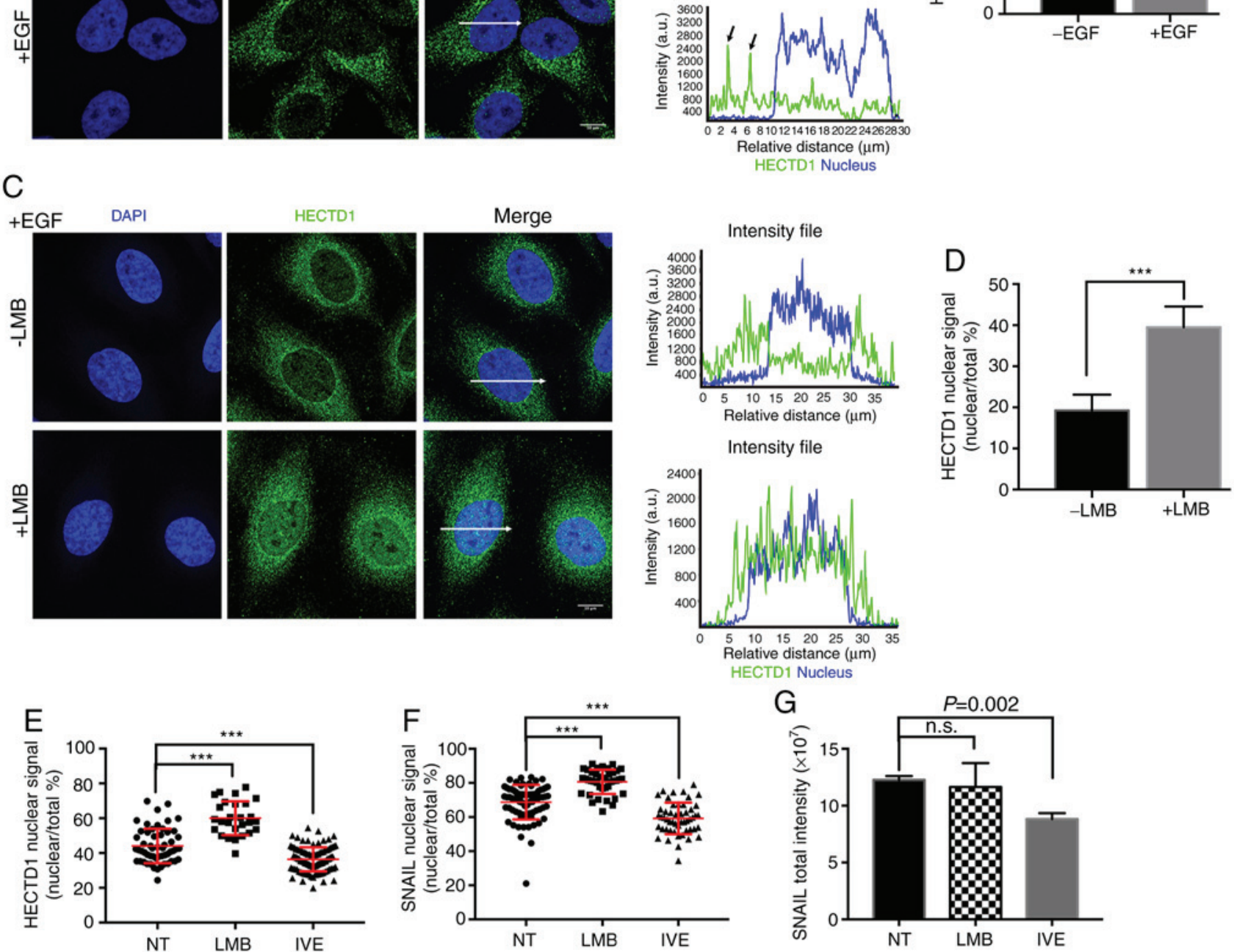

Figure 3. HECTD1 translocates between the nucleus and cytoplasm. (A) HECTD1 shuttles between the nucleus and cytoplasm with or without EGF treatment. The subcellular localization of HECTD1 was analyzed using fluorescence microscopy. HeLa cells were treated with or without $100 \mathrm{ng} / \mathrm{ml} \mathrm{EGF}$ for $6 \mathrm{~min}$ and immunostained with anti-HECTD1 antibody. The nucleus was stained with DAPI. The arrow-lines indicate the subcellular localizations of SNAIL, measured as the fluorescent intensity in individual cells. (B) The export of HECTD1 from the nucleus is sensitive to LMB treatment. HeLa cells were treated with or without $50 \mathrm{nM} \mathrm{LMB}$ for $4 \mathrm{~h}$ and sequentially treated with $100 \mathrm{ng} / \mathrm{ml} \mathrm{EGF}$. Cells were stained with anti-HECTD1 antibody and the fluorescent intensity demonstrates HECTD1 localization. Scale bar, $10 \mu \mathrm{m}$. (C and D) Intensity of HECTD1 nuclear signals and whole cells were analyzed and semi-quantified, and the percentage of nuclear signals are presented. Data were analyzed by the Student's t-test and presented as the means \pm SD. ${ }^{* * *} \mathrm{P}<0.001$. LMB, leptomycin-B; EGF, epidermal growth factor; HECTD1, HECT domain E3 ubiquitin ligase 1. Nuclear signals of (E) HECTD1 and (F) SNAIL were analyzed in wild-type Hela cells with not treatment (NT), or with LMB $(50 \mathrm{nM})$ or IVE $(1 \mu \mathrm{M})$. The percentages of cell nuclear signal are shown. Statistical analysis was performed using one-way ANOVA with Tukey's multiple comparisons post hoc test. The data are shown as the means \pm SD. ${ }^{* * *} \mathrm{P}<0.001$. (G) Quantification of SNAIL total signal intensities of wild-type cells were analyzed in different treatments. The data analyzed by one-way ANOVA and are shown as the means \pm SD. n.s., no significant differences. More than 30 cells in each treatment condition were measured.

that the EGF-receptor (EGFR) is overexpressed in cervical cancer (25), and SLUG/SNAIL are downstream mediators of EGFR-stimulated re-epithelization (26). Thus, the study investigated whether the protein expression levels of several important factors in EGF-mediated EMT were associated with the knockdown of HECTD1 expression. The Ctrl HeLa cells expressed low levels of SNAIL, whereas the HECTD1-KD cells demonstrated high expression levels of SNAIL (Figs. 4D and S4A). Similar results were found for SLUG. The adhesion molecule, E-cadherin, is considered to be an important signaling marker for EMT (27); reduced E-cadherin expression levels were observed in the HECTD1-KD cells, 
A

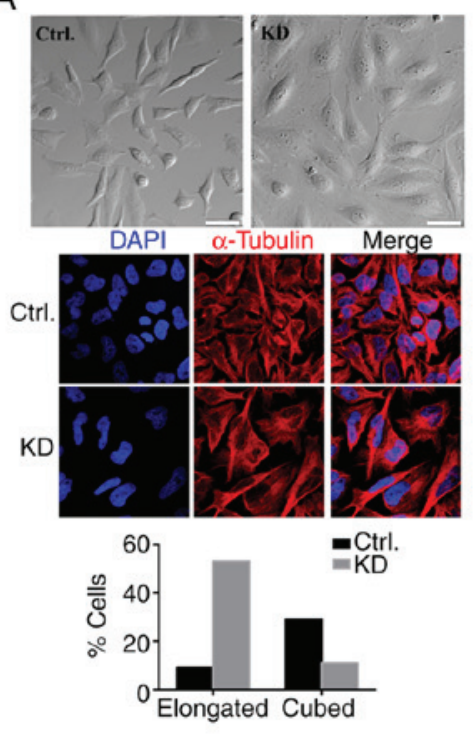

B
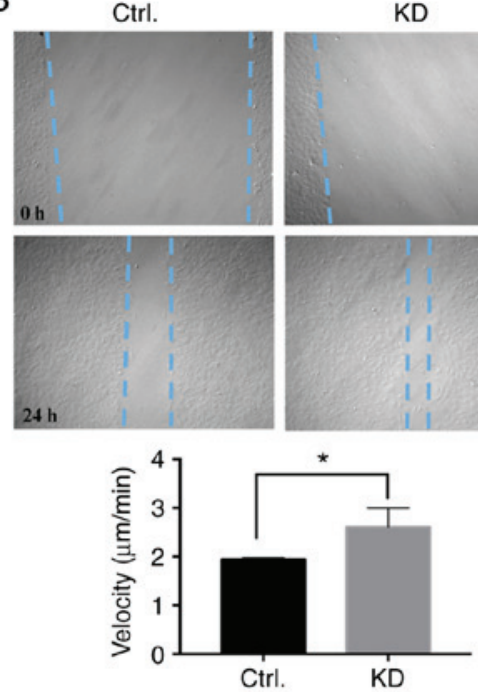

C
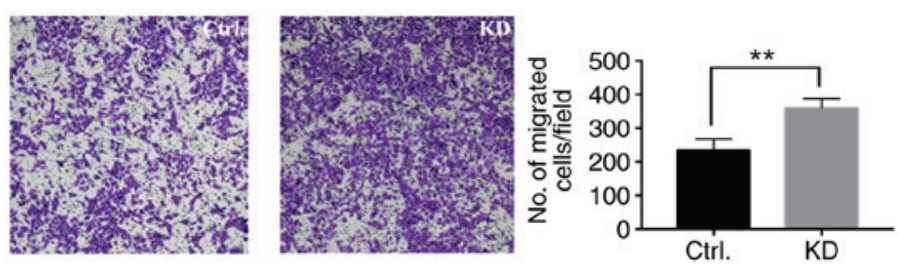

D
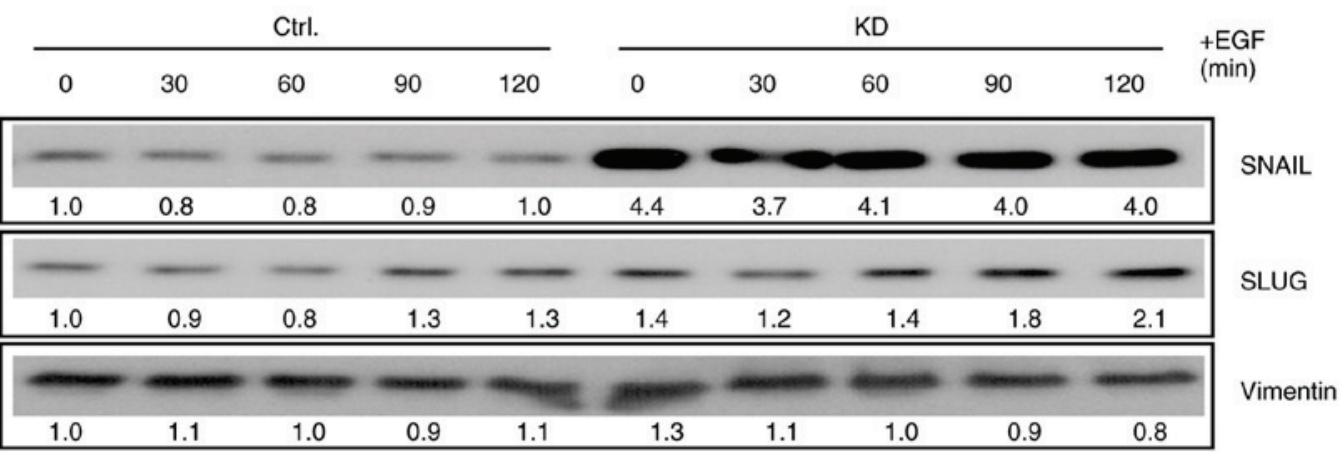

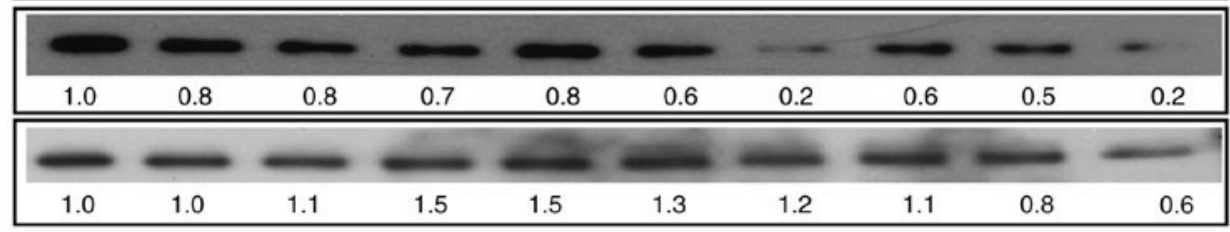

E-cadherin

$\mathrm{N}$-cadherin

GAPDH

Figure 4. Knockdown of HECTD1 potentiates EMT. (A) Morphological changes in HeLa cells following HECTD1 knockdown with short hairpin RNA. HeLa cells transfected with Ctrl or HECTD1-KD exhibited an elongated and highly multipolar morphology. In the lower panel, cells were immunostained with an anti- $\alpha$-tubulin antibody. Scale bar, $50 \mu \mathrm{m}$. Percentage of spindle-like phenotype (elongated) over 'cobblestone' phenotype (cubed) of cells were determined in a single experiment. (B) Wound healing assays were performed on fibronectin for $24 \mathrm{~h}$ in medium containing $1 \mu \mathrm{M}$ aphidicolin and cell migration was semi-quantified. Statistical analysis was performed using the Student's t-test. " $\mathrm{P}<0.05$. (C) Transwell migration assays were performed in HECTD1-KD-transfected (FI316530) Ca Ski cells. Results obtained from a single knockdown are presented. Cells in 4 randomly selected fields were counted using a microscope. Statistical analysis was performed using the Student's t-test. ${ }^{* *} \mathrm{P}<0.01$. (D) Western blot analysis for selected EMT markers in Ctrl- or HECTD1-KD-transfected cells following EGF treatment. HeLa cells were treated with $100 \mathrm{ng} / \mathrm{ml}$ EGF for 0-120 min following overnight serum starvation. The numbers found below each lane indicate the quantified protein expression following normalization with GAPDH. Ctrl, negative control, HECTD1, HECT domain E3 ubiquitin ligase 1; KD, knockdown; EMT, epithelial-mesenchymal transition; EGF, epidermal growth factor.

which indicated that these cells had a lower cell adhesive ability. The expression level of vimentin remained unaltered, while that of $\mathrm{N}$-cadherin was found to be slightly decreased. Overall, these data suggested that the knockdown of HECTD1 may potentiate EMT.
Knockdown of HECTDI sustains EGF signaling. In cervical cancer cells, EGF has been reported to induce EMT through the upregulation of the expression of SNAIL (28) and via the activation of the AKT and ERK signaling pathways (29). Furthermore, hypoxia activates the EGFR signaling pathway 
to induce EMT (30). In this study, to determine the changes in the EGFR-signaling pathway following stimulation of cells with EGF, total ERK1/2 and phospho-ERK1/2 protein expression levels were investigated by western blot analysis at different time points (Fig. 5). Compared with the Ctrl cells, the expression levels of total ERK1/2 were not markedly altered in the HECTD1-KD cells (Figs. 5A and S4B). However, the phosphorylation of ERK1/2 progressively decreased following a 30-min incubation period with EGF in the Ctrl cells, whereas the phospho-ERK1/2 expression levels remained high in the HECTD1-KD cells at 60 min following EGF stimulation (Fig. 5A and B). In addition, the knockdown of HECTD1 increased cell motility (Fig. 4B and C) and EGFR signaling was observed to be associated with an increased phospho-ERK1/2 expression (Fig. 5A), suggesting that the knockdown of HECTD1 and the EGF signaling pathway may synergize to promote EMT in these cells. It was also particularly intriguing to observe the reduction of total ERK2 protein levels in the HECTD1-KD cells.

Expression of HECTD1 is regulated by miR-210 under hypoxic conditions. Upon screening for factors that regulated the expression levels of Hectdl in mouse embryonic fibroblasts cells, Hectdl expression was observed to be downregulated during hypoxic and heat treatment (11). HECTD1 is a putative BH3-only protein under hypoxic conditions (12); it has been reported that hypoxia is involved in the EMT processes (31), and that both miR-21 and miR-210 are also involved in this process $(32,33)$. In this study, miRTarBase was used to identify hypothetical target sequences for HECTD1. Six targeting sequences of mir-21 and mir-210 were found in the 3 'UTR of HECTD1. Thus, HECTD1 may be a target of miR-21 and miR-210 (34). By inducing hypoxic conditions in cells using $\mathrm{CoCl}_{2}$, a factor involved in inducing EMT in cancer, the association between the expression levels of HECTD1 and those of miR-21 and miR-210 were investigated. The expression levels of $H E C T D 1$ progressively decreased under hypoxic conditions in HeLa cervical cancer cells (Fig. 6A); however, the expression levels of miR-21 and miR-210 were increased in the HeLa cells following $\mathrm{CoCl}_{2}$ treatment (Fig. 6A).

The involvement of miR-21/-210 in the inhibition of HECTD1 expression was further investigated by transfecting the cells with their specific antagomirs ( $\alpha$-mir) (35). The presence of the mir-210 antagomir compared with the scramble $\left(\alpha\right.$-scr), followed by $\mathrm{CoCl}_{2}$ treatment, resulted in significantly increased mRNA expression levels of HECTDI (Fig. 6B; P<0.001). Similar results were obtained with miR-21 antagomirs, although to a lesser extent.

HECTD1 expression is positively associated with clinical outcome. To determine whether the genetic abnormalities of HECTD1 expression may affect its function in both cervical cancer cells and in other types of cancer cells, 329 cervical cancer samples from The Catalogue Of Somatic Mutations In Cancer (COSMIC) (36) and 309 samples from The Cancer Genome Atlas (TGCA) were analyzed using whole-exome sequencing (37). A total of 24 mutations were identified throughout the HECTD1 gene, including one frameshift, twenty missense and three silent mutations (Fig. 7A, and Tables SI and SII). Although all these variants were spread over the entire gene, and they occurred most commonly in those areas
A
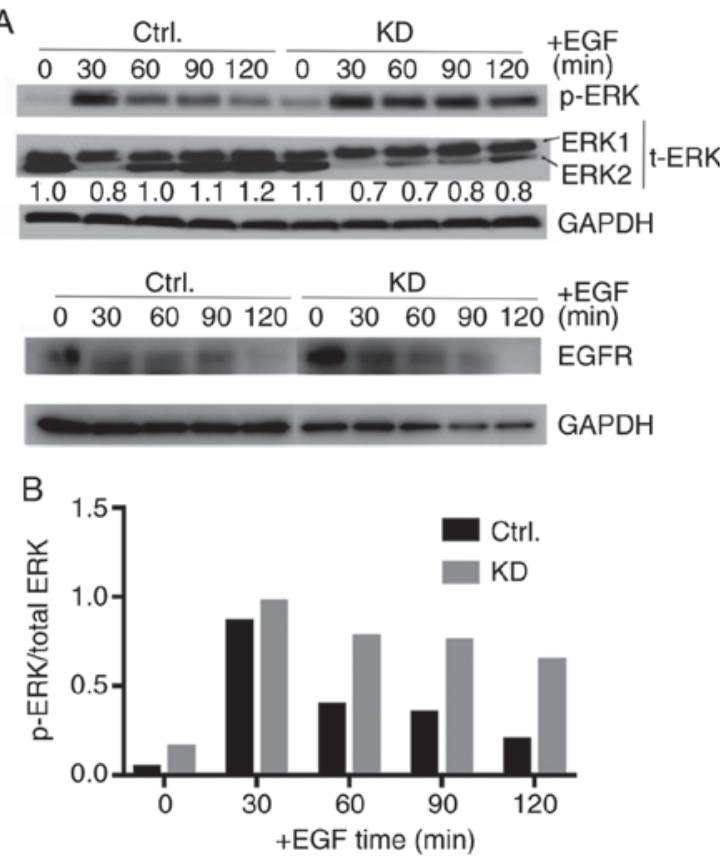

Figure 5. EGF signaling pathway is affected following EGF stimulation. (A) T-ERK1/2 and phospho-ERK1/2 (Thr202/Tyr204) were detected following EGF treatment. t-ERK protein expression was semi-quantified and normalized to GAPDH expression. The lower panel represents the results from a repeated experiment to determine the expression of EGFR. (B) Semi-quantitative analysis of results obtained from part A in a single experiment. phospho-ERK1/2 protein expression levels were normalized to t-ERK expression levels. t, total; EGF, epidermal growth factor.

which encoded the evolutionally conserved ANK-repeats, the heavily phosphorylated area and the HECT-domain (Fig. 7A). Compared with other types of cancer tissue, such as ovarian cancer, these genetic abnormalities were exclusively observed in small cell carcinoma, squamous cell carcinoma and endocervical adenocarcinoma (Fig. 7A, and Tables SI and SIII).

Using tissue microarrays, the association between the expression of HECTD1 and SNAIL in cervical cancer samples was investigated. A significant inverse association was observed between the nuclear expression of HECTD1 and SNAIL; all 4 samples displayed a low HECTD1 expression in the presence of a high SNAIL expression (Figs. 7B and S5).

Moreover, Kaplan-Meier analysis of squamous cell carcinoma using the HPA (38) revealed that markedly higher HECTD1 expression levels were associated with a longer relapse-free survival $\left(\mathrm{P}=4.86 \times 10^{-2}\right.$; Fig. $\left.7 \mathrm{C}\right)$. By contrast, higher SNAIL expression levels were associated with a shorter relapse-free survival $\left(\mathrm{P}=2.25 \times 10^{-2}\right)$. The association between HECTD1 expression in cancer tissue samples and the survival rate in a number of large public clinical databases was also investigated (39). In breast, gastric, lung and kidney cancer, lower expression levels of HECTD1 were significantly associated with shorter survival times for patients with cancer (Fig. S6). Furthermore, consistent with the role of miR-210 in regulating HECTD1 expression (Fig. 6), higher expression levels of miR-210 were associated with lower expression levels of HECTD1 and a shorter patient survival (Fig. S7), which is similar to results obtained in cervical cancer, where it was reported that the upregulation of miR-210 was associated with a poorer prognosis (40). 

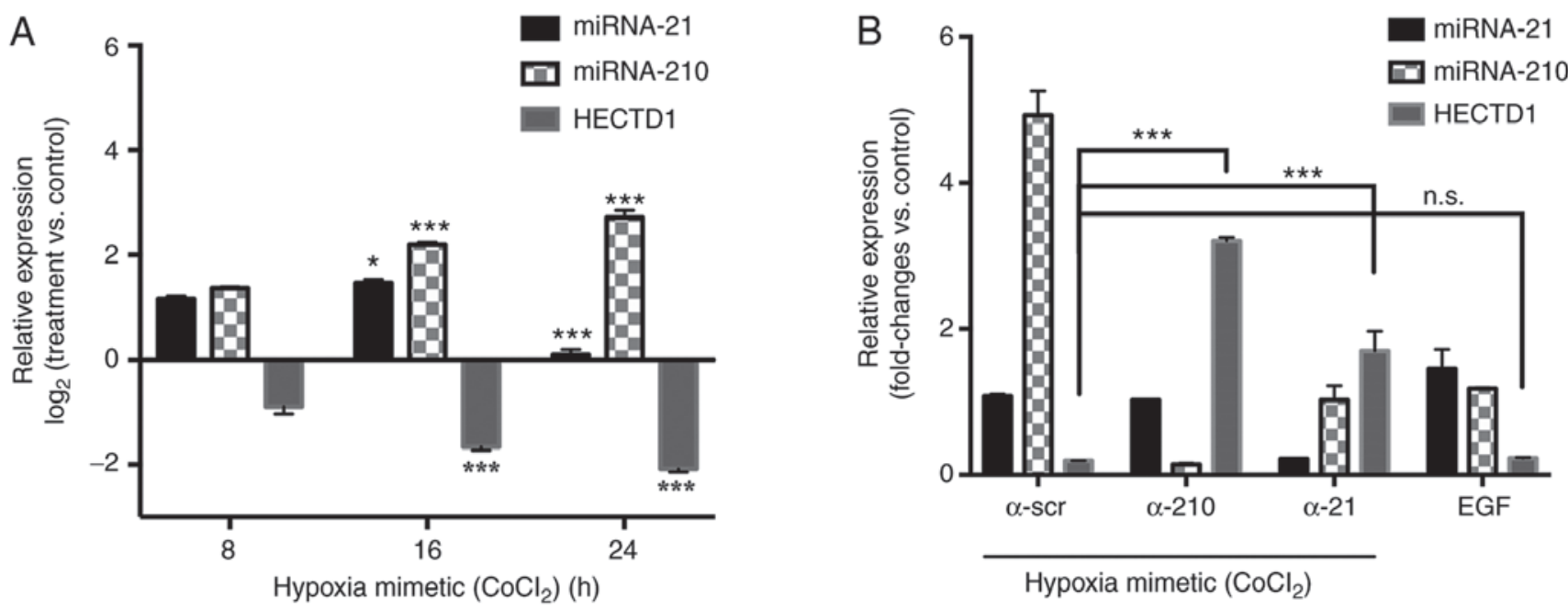

Figure 6. Regulation of HECTD1 expression levels by miR-210 under hypoxic conditions. (A) HeLa cells were treated with $500 \mu \mathrm{M} \mathrm{CoCl}$ to induce hypoxic conditions for the indicated time periods. RT-qPCR was performed to determine the expression levels of mature miR-21/210 and HECTD1. Each value was normalized to U6 expression levels and is expressed relative to the expression levels of miR-21/miR-210 in HeLa cells treated with the Ctrl. Data were analyzed by one-way ANOVA at $8 \mathrm{~h}$ and are presented as the means \pm SEM of 3 independent experimental repeats. ${ }^{*} \mathrm{P}<0.05,{ }^{* * * *} \mathrm{P}<0.001$. Relative expression $\left[\log _{2}(\right.$ treatment/control)] was quantified by normalizing values to expression levels recorded at $0 \mathrm{~h}$. (B) Effect of the antagomir miR-21/210 transfection in HeLa cells on HECTD1 expression using RT-qPCR. HeLa cells were transfected with miR-21 or miR-210 antagomir or Ctrl for $16 \mathrm{~h}$. Other cells were treated with $500 \mu \mathrm{M}$ $\mathrm{CoCl}_{2}$ or $100 \mathrm{ng} / \mathrm{ml}$ EGF for $16 \mathrm{~h}$. Expression levels were quantified by normalizing expression levels to cells not exposed to CoCl $\mathrm{Cr}_{\mathrm{EGF}}$. Statistical analysis was performed using unpaired one-way ANOVA. ${ }^{* * *} \mathrm{P}<0.001$. RT-qPCR, reverse transcription-quantitative PCR; HECTD1, HECT domain E3 ubiquitin ligase 1; Ctrl, negative control; n.s., not significant; miR, microRNA; EGF, epidermal growth factor.

\section{Discussion}

Previously, Zohn et al (41) and our previous research (11) reported that Hectdl-homozygous mutant embryos display numerous defects during their development and HECTD1 has been observed to interact with proteins involved in several distinct signaling pathways, indicating that HECTD1 may target numerous cellular processes. The present study demonstrated that HECTD1 interacted with SNAIL and promoted its HECTD1-mediated ubiquitination, suggesting that HECTD1 may be one of the numerous E3 ubiquitin ligases regulating the protein stability of SNAIL. Thus, the effects of HECTD1 on SNAIL protein stability and degradation suggested that HECTD1 may be functionally important during tumorigenesis under certain conditions of cellular stress.

The nuclear import and export of HECTD1 and SNAIL are tightly regulated; HECTD1 contains several putative NLS and NES and it has been reported that HECTD1 was pulled-down and co-immunoprecipitated with Importin $\alpha 3 / 7$ (42), suggesting that HECTD1 is imported into the nucleus via classical nuclear protein transport mechanisms. In the present study, LMB treatment was observed to inhibit the nuclear export of HECTD1, indicating that the nuclear export protein, $\mathrm{XPO1/CRM1}$, is involved in regulating the nuclear retention of HECTD1. It was also demonstrated that HECTD1 interacted with SNAIL and that the cellular localization of SNAIL was paradoxical to HECTD1 following EGF stimulation. Notably, it was observed that the overexpression of SNAIL inhibited HECTD1 expression. SNAIL is a transcriptional factor that exerts global effects on gene expression; it can repress gene expression by using a SNAG domain, a CtBP binding motif or by directly recruiting repressor complexes, thus it is possible that HECTD1 is a SNAIL target gene. The accumulation of SNAIL proteins in the nucleus following HECTD1-KD suggested that that the degradation of SNAIL via ubiquitination may occur in the nucleus or that SNAIL translocates to the cytoplasm, where its degradation is subsequently mediated by HECTD1. The nuclear retention of HECTD1 following LMB treatment did not reduce the expression levels of SNAIL; however, the fact that the IVE-induced accumulation of HECTD1 in the cytoplasm induced the degradation of SNAIL favors the later possibility. In agreement with these observations, a recent study (43) used cellular fractionation and western blot analysis to demonstrate that SNAIL was localized in both the cytosol and the nucleus, and the nuclear localization of SNAIL was reduced following the serum starvation of the cells. Of note, it was also observed that the overexpression of SNAIL inhibited HECTD1 expression. It is possible that HECTD1 is a transcriptional target of SNAIL. Thus, future experiments with targeted mutagenesis to all 4 NESs in the ANK repeat region may clarify the mechanisms through which HECTD1 ubiquitinates SNAIL. Previously, several E3-ubiquitin ligases have been reported to play crucial roles in the regulation of SNAIL degradation (19-21). The finding of this study that HECTD1 is a novel E3 ligase for SNAIL provides new insight into the regulation of EMT by E3-ubiquitin ligases.

The knockdown of HECTD1 markedly induced changes in the mesenchymal phenotype of cells, including cellular morphology and migration, and increased the expression levels of SNAIL. In cervical cancer cells, EGF treatment induced EMT, which was associated with the increased expression of EGFR and disease progression (28); however, in the present study, the knockdown of HECTD1 did not significantly modify EGFR expression upon stimulation with EGF, but instead regulated the protein expression levels of SNAIL. Furthermore, EGF treatment induced the nuclear export of HECTD1, which increased SNAIL expression levels in the nucleus. These results indicated that the promotion of EMT 
A

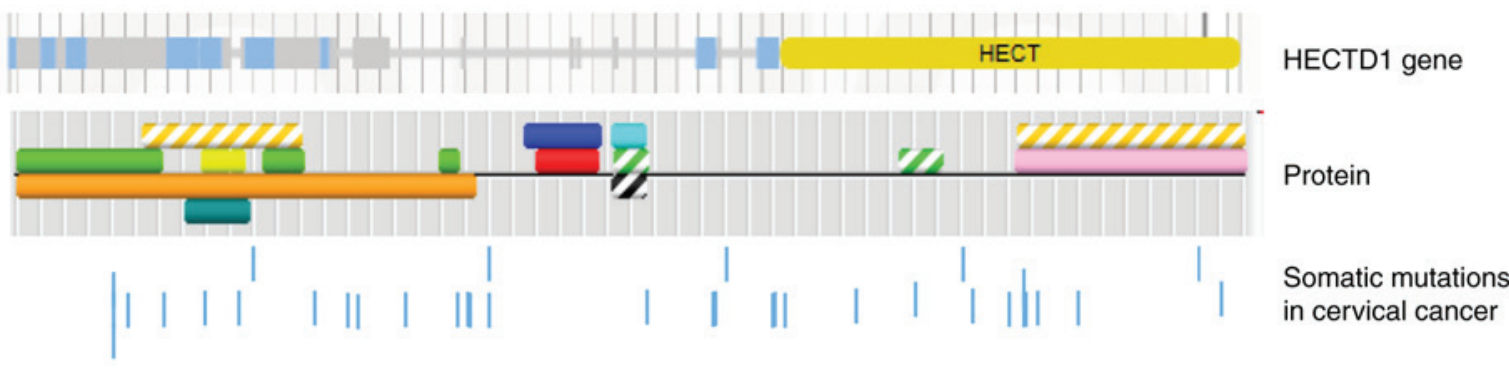

B
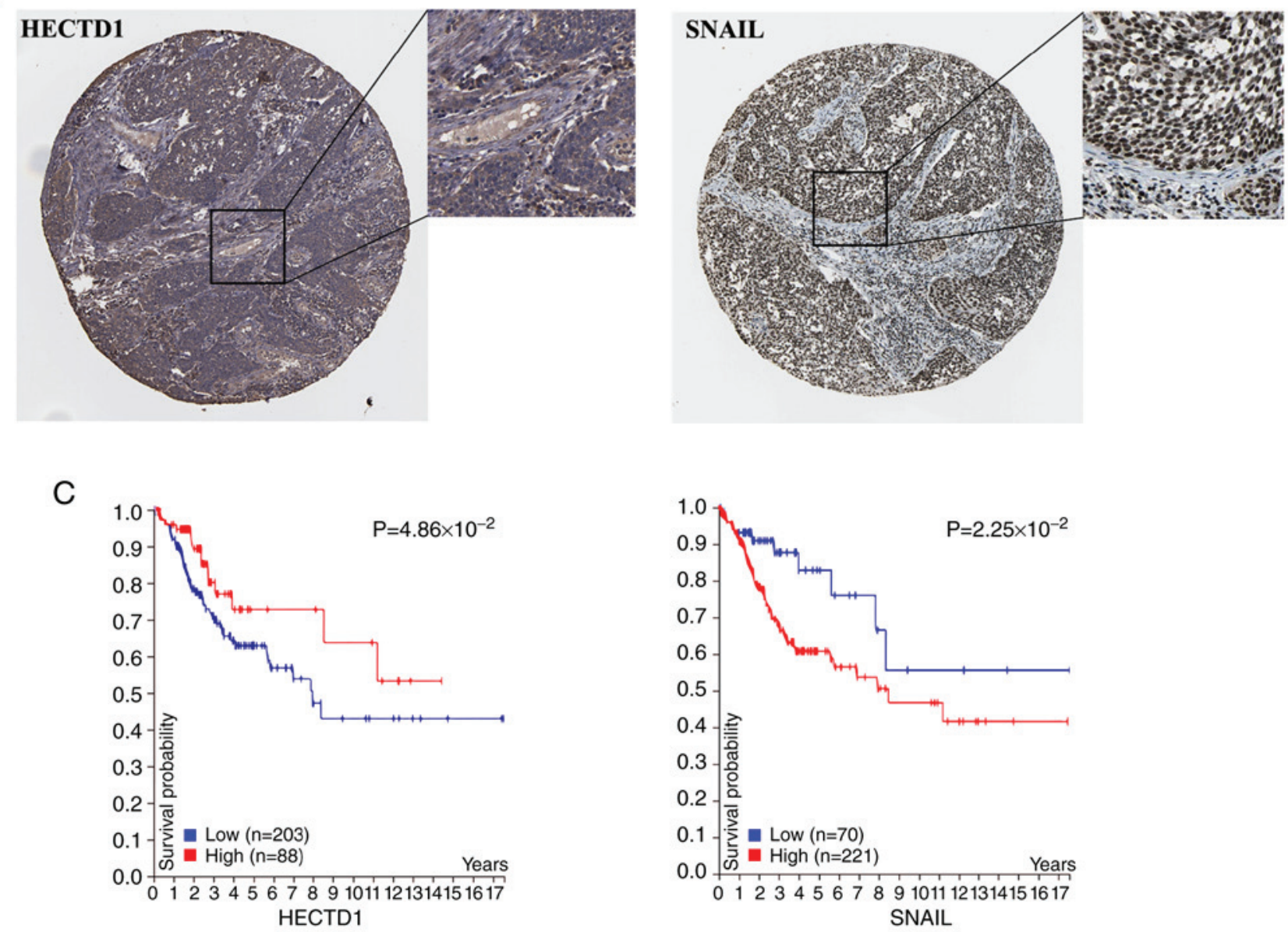

Figure 7. Analysis of the association of HECTD1 expression levels with clinical outcome in cervical cancer. (A) Schematic representation of the HECTD1 gene and the positions of somatic mutations identified from The Cancer Genome Atlas. (B) Representative immunohistochemical images of HECTD1 (left panel) and SNAIL (right panel) protein expression levels in cervical cancer obtained from the HPA. (C) Low HECTD1 expression levels were associated with a poor survival of patients with cervical cancer. Kaplan-Meier plots presenting the relapse-free survival of cohorts of patients from the HPA. HECTD1 expression levels in patients is negatively correlated with SNAIL expression levels. HPA, Human Protein Atlas; HECTD1, HECT domain E3 ubiquitin ligase 1.

by the EGFR-mediated signaling pathway may be modulated by HECTD1 downstream of EGF signaling. It has previously reported that HECTD1 is phosphorylated by EGF $(44,45)$; thus, future studies are warranted to investigate whether the phosphorylation of HECTD1 interferes with its nuclear localization.

Under hypoxic conditions, in which $\mathrm{CoCl}_{2}$ was used to induce cellular responses mediated by hypoxia (46), HECTD1 expression levels decreased, which is consistent with the reduced protein expression levels observed under hypoxia (47). Furthermore, the nuclear translocation of the SNAIL protein increased when cells were exposed to hypoxia (48). These data suggest that the knockdown of HECTD1 expression by hypoxia, or even non-hypoxic conditions that induce HIF-1 $\alpha$ stabilization or deregulation of miRNAs, may therefore increase the protein expression levels of SNAIL and downregulate those of E-cadherin, thereby promoting EMT.

The involvement of HECTD1 in EMT in HeLa cells was surprising, since HECTD1-KD cells exhibited no differences in their growth rate compared with the Ctrl. These observations suggested that the selection of HECTD1-KD cells may result in a more aggressive metastasis-like phenotype of malignant cells. Consistent with this hypothesis, the decreased expression levels of HECTD1 were observed to be significantly associated 
with a poor patient survival, not only in cervical cancer, but also in breast and gastric cancer. However, the present study used a small number of patient samples; therefore, further investigations using larger cohorts may provide further insight into the role of HECTD1 in cancer.

Notably, a recent study by Duhamel et al (49) observed that the HECTD1-mediated regulation of ACF7, a +TIPs protein required for cell migration, modulated EMT during metastasis in breast cancer; the decreased expression of HECTD1 promoted ACF7-induced EMT, invasion and metastasis. Thus, the results of the present study, alongside the results from the study by Duhamel et al (49), suggested that HECTD1 may promote EMT through multiple different mechanisms by targeting distinct molecules.

In conclusion, HECTD1 may be one of the E3 ubiquitin ligases that controls the stability of SNAIL proteins, and it may mediate EMT in cervical cancer by regulating cell migration and the expression of transcription factors, such as SNAIL.

\section{Acknowledgements}

The authors are grateful to Dr Michael Abanto (University of Basel) in supporting our work with the confocal microscope. The authors are also grateful to Dr Anne-Catherine Feutz (University of Basel) for her comments and language editing of the manuscript. In addition, the authors would like to thank Ms. Hedwig Wariwoda (University of Basel) for her assistance with the LMB assay.

\section{Funding}

This study was supported by the University of Basel. This study was also funded by the Repronatal Foundation, Basel, Switzerland. XW is the recipient of the China Scholarship Council Stipendium of P.R. China.

\section{Availability of data and materials}

All data generated or analyzed during the present study are included in this article and its supplementary files.

\section{Authors' contributions}

HZ, CDG, XW and ZJ conceived and designed the experiments presented in this manuscript. YP performed the $\mathrm{CHX}$ chase assay. XW, HZ and CDG wrote the manuscript. All authors discussed the results, and read and approved the final manuscript.

\section{Ethics approval and consent to participate}

Not applicable.

\section{Patient consent for publication}

Not applicable.

\section{Competing interests}

The authors declare that they have no competing interests.

\section{References}

1. Sahu SK, Tiwari N, Pataskar A, Zhuang Y, Borisova M, Diken M, Strand S, Beli P and Tiwari VK: FBXO32 promotes microenvironment underlying epithelial-mesenchymal transition via CtBP1 during tumour metastasis and brain development. Nat Commun 8: 1523, 2017.

2. Liu Y, Zhou H, Zhu R, Ding F, Li Y, Cao X and Liu Z: SPSB3 targets SNAIL for degradation in GSK-3beta phosphorylation-dependent manner and regulates metastasis. Oncogene 37: 768-776, 2018

3. Park SH, Jung EH, Kim GY, Kim BC, Lim JH and Woo CH: Itch E3 ubiquitin ligase positively regulates TGF- $\beta$ signaling to EMT via smad7 ubiquitination. Mol Cells 38: 20-25, 2015.

4. Van Laar RK: Genomic signatures for predicting survival and adjuvant chemotherapy benefit in patients with non-small-cell lung cancer. BMC Med Genomics 5: 30, 2012.

5. Lim SK, Lu SY, Kang SA, Tan HJ, Li Z, Adrian Wee ZN, Guan JS, Reddy Chichili VP, Sivaraman J, Putti T, et al: Wnt signaling promotes breast cancer by blocking ITCH-Mediated degradation of YAP/TAZ transcriptional coactivator WBP2. Cancer Res 76: 6278-6289, 2016.

6. Zhou H, Liu Y, Zhu R, Ding F, Wan Y, Li Y and Liu Z: FBXO32 suppresses breast cancer tumorigenesis through targeting KLF4 to proteasomal degradation. Oncogene 36: 3312-3321, 2017.

7. Komander D: The emerging complexity of protein ubiquitination. Biochem Soc Trans 37: 937-953, 2009.

8. Pickart CM and Eddins MJ: Ubiquitin: Structures, functions, mechanisms. Biochim Biophys Acta 1695: 55-72, 2004.

9. Metzger MB, Hristova VA and Weissman AM: HECT and RING finger families of E3 ubiquitin ligases at a glance. J Cell Sci 125: 531-537, 2012.

10. Scheffner M and Kumar S: Mammalian HECT ubiquitin-protein ligases: Biological and pathophysiological aspects. Biochim Biophys Acta 1843: 61-74, 2014.

11. D'Alonzo D, Emch FH, Shen X, Bruder E, De Geyter C and Zhang H: Hectd1 is essential for embryogenesis in mice. Gene Expr Patterns 10: 119064, 2019.

12. Shen X, Jia Z, D'Alonzo D, Wang X, Bruder E, Emch FH, De Geyter $\mathrm{C}$ and Zhang H: HECTD1 controls the protein level of IQGAP1 to regulate the dynamics of adhesive structures. Cell Commun Signal 15: 2, 2017.

13. Zhu S, Si ML, Wu H and Mo YY: MicroRNA-21 targets the tumor suppressor gene tropomyosin 1 (TPM1). J Biol Chem 282: 14328-14336, 2007.

14. Besnier M, Gasparino S, Vono R, Sangalli E, Facoetti A, Bollati V, Cantone L, Zaccagnini G, Maimone B, Fuschi P, et al: MiR-210 enhances the therapeutic potential of bone-marrow-derived circulating proangiogenic cells in the setting of limb ischemia. Mol Ther 26: 1694-1705, 2018.

15. Fan Q, Hu X, Zhang H, Wang S, Zhang H, You C, Zhang CY, Liang H, Chen X and Ba Y: MiR-193a-3p is an important tumour suppressor in lung cancer and directly targets KRAS. Cell Physiol Biochem 44: 1311-1324, 2017.

16. Livak KJ and Schmittgen TD: Analysis of relative gene expression data using real-time quantitative PCR and the 2(-Delta Delta C(T)) method. Methods 25: 402-408, 2001.

17. Kaundal R and Raghava GP: RSLpred: An integrative system for predicting subcellular localization of rice proteins combining compositional and evolutionary information. Proteomics 9: 2324-2342, 2009.

18. Nakai K and Horton P: PSORT: A program for detecting sorting signals in proteins and predicting their subcellular localization. Trends Biochem Sci 24: 34-36, 1999.

19. Vinas-Castells R, Frias A, Robles-Lanuza E, Zhang K, Longmore GD, García de Herreros A and Díaz VM: Nuclear ubiquitination by FBXL5 modulates Snail1 DNA binding and stability. Nucleic Acids Res 42: 1079-1094, 2014.

20. Zheng H, Shen M, Zha YL, Li W, Wei Y, Blanco MA, Ren G, Zhou T, Storz P, Wang HY and Kang Y: PKD1 phosphorylation-dependent degradation of SNAIL by SCF-FBXO11 regulates epithelial-mesenchymal transition and metastasis. Cancer Cell 26: 358-373, 2014.

21. Zhou BP, Deng J, Xia W, Xu J, Li YM, Gunduz M and Hung MC: Dual regulation of snail by GSK-3beta-mediated phosphorylation in control of epithelial-mesenchymal transition. Nat Cell Biol 6: 931-940, 2004.

22. Hutten S and Kehlenbach RH: CRM1-mediated nuclear export: To the pore and beyond. Trends Cell Biol 17: 193-201, 2007. 
23. Wolff B, Sanglier JJ and Wang Y: Leptomycin B is an inhibitor of nuclear export: Inhibition of nucleo-cytoplasmic translocation of the human immunodeficiency virus type 1 (HIV-1) Rev protein and Rev-dependent mRNA. Chem Biol 4: 139-147, 1997.

24. Chaudhry P, Fabi F, Singh M, Parent S, Leblanc V and Asselin E: Prostate apoptosis response- 4 mediates TGF- $\beta$-induced epithelial-to-mesenchymal transition. Cell Death Dis 5: e1044, 2014.

25. Schrevel M, Gorter A, Kolkman-Uljee SM, Trimbos JB, Fleuren GJ and Jordanova ES: Molecular mechanisms of epidermal growth factor receptor overexpression in patients with cervical cancer. Mod Pathol 24: 720-728, 2011.

26. Kusewitt DF, Choi C, Newkirk KM, Leroy P, Li Y, Chavez MG and Hudson LG: Slug/Snai2 is a downstream mediator of epidermal growth factor receptor-stimulated reepithelialization. J Invest Dermatol 129: 491-495, 2009.

27. Zeisberg M and Neilson EG: Biomarkers for epithelial-mesenchymal transitions. J Clin Invest 119: 1429-1437, 2009.

28. LeeMY,Chou CY,Tang MJandShenMR:Epithelial-Mesenchymal transition in cervical cancer: Correlation with tumor progression, epidermal growth factor receptor overexpression, and snail up-regulation. Clin Cancer Res 14: 4743-4750, 2008.

29. Gan $Y$, Shi $C$, Inge $L$, Hibner $M$, Balducci J and Huang $Y$ : Differential roles of ERK and Akt pathways in regulation of EGFR-mediated signaling and motility in prostate cancer cells. Oncogene 29: 4947-4958, 2010.

30. Misra A, Pandey C, Sze SK and Thanabalu T: Hypoxia activated EGFR signaling induces epithelial to mesenchymal transition (EMT). PLoS One 7: e49766, 2012.

31. Lamouille S, Xu J and Derynck R: Molecular mechanisms of epithelial-mesenchymal transition. Nat Rev Mol Cell Biol 15: 178-196, 2014

32. Zavadil J, Narasimhan M, Blumenberg M and Schneider RJ: Transforming growth factor-beta and microRNA:MRNA regulatory networks in epithelial plasticity. Cells Tissues Organs 185: 157-161, 2007.

33. Ding L, Zhao L, Chen W, Liu T, Li Z and Li X: MiR-210, a modulator of hypoxia-induced epithelial-mesenchymal transition in ovarian cancer cell. Int J Clin Exp Med 8: 2299-2307, 2015.

34. Fasanaro P, Greco S, Lorenzi M, Pescatori M, Brioschi M, Kulshreshtha R, Banfi C, Stubbs A, Calin GA and Ivan M: An integrated approach for experimental target identification of hypoxia-induced miR-210. J Biol Chem 284: 35134-35143, 2009.

35. Cicchillitti L, Di Stefano V, Isaia E, Crimaldi L, Fasanaro P, Ambrosino V, Antonini A, Capogrossi MC, Gaetano C, Piaggio $\mathrm{G}$ and Martelli F: Hypoxia-Inducible factor $1-\alpha$ induces miR-210 in normoxic differentiating myoblasts. J Biol Chem 287: 44761-44771, 2012.

36. Forbes SA, Tang G, Bindal N, Bamford S, Dawson E, Cole C, Kok CY, Jia M, Ewing R, Menzies A, et al: COSMIC (the Catalogue of Somatic Mutations in Cancer): A resource to investigate acquired mutations in human cancer. Nucleic Acids Res 38: D652-D657, 2010.
37. Tomczak K, Czerwinska $\mathrm{P}$ and Wiznerowicz M: The cancer genome atlas (TCGA): An immeasurable source of knowledge. Contemp Oncol (Pozn) 19: A68-A77, 2015.

38. Uhlen M, Zhang C, Lee S, Sjöstedt E, Fagerberg L, Bidkhori G, Benfeitas R, Arif M, Liu Z, Edfors F, et al: A pathology atlas of the human cancer transcriptome. Science 357: 6352, 2017.

39. Lanczky A, Nagy A, Bottai G, Munkácsy G, Szabó A, Santarpia L and Gyórffy B: MiRpower: A web-tool to validate survival-associated miRNAs utilizing expression data from 2178 breast cancer patients. Breast Cancer Res Treat 160: 439-446, 2016.

40. Rao Q, Shen Q, Zhou H, Peng Y, Li J and Lin Z: Aberrant microRNA expression in human cervical carcinomas. Med Oncol 29: 1242-1248, 2012.

41. Zohn IE, Anderson KV and Niswander L: The hectd1 ubiquitin ligase is required for development of the head mesenchyme and neural tube closure. Dev Biol 306: 208-221, 2007.

42. Hugel S, Depping R, Dittmar G, Rother F, Cabot R, Sury MD, Hartmann $\mathrm{E}$ and Bader M: Identification of importin $\alpha 7$ specific transport cargoes using a proteomic screening approach. Mol Cell Proteomics 13: 1286-1298, 2014.

43. Zada S, Hwang JS, Ahmed M, Lai TH, Pham TM and Kim DR: Control of the epithelial-to-mesenchymal transition and cancer metastasis by autophagy-dependent SNAI1 Degradation. Cells 8: E129, 2019.

44. Liu G, Rogers J, Murphy CT and Rongo C: EGF signalling activates the ubiquitin proteasome system to modulate $\mathrm{C}$. elegans lifespan. EMBO J 30: 2990-3003, 2011.

45. Argenzio E, Bange T, Oldrini B, Bianchi F, Peesari R, Mari S, Di Fiore PP, Mann M and Polo S: Proteomic snapshot of the EGF-induced ubiquitin network. Mol Syst Biol 7: 462, 2011.

46. Piret JP, Mottet D, Raes M and Michiels C: $\mathrm{CoCl} 2$, a chemical inducer of hypoxia-inducible factor-1, and hypoxia reduce apoptotic cell death in hepatoma cell line HepG2. Annals N Y Acad Sci 973: 443-447, 2002.

47. Djidja MC, Chang J, Hadjiprocopis A, Schmich F, Sinclair J, Mršnik M, Schoof EM, Barker HE, Linding R, Jørgensen C and Erler JT: Identification of hypoxia-regulated proteins using MALDI-mass spectrometry imaging combined with quantitative proteomics. J Proteome Res 13: 2297-2313, 2014.

48. Lester RD, Jo M, Montel V, Takimoto S and Gonias SL: UPAR induces epithelial-mesenchymal transition in hypoxic breast cancer cells. J Cell Biol 178: 425-436, 2007.

49. Duhamel S, Goyette MA, Thibault MP, Filion D, Gaboury L and Cote JF: The E3 ubiquitin ligase HectD1 suppresses EMT and metastasis by targeting the +TIP ACF7 for degradation. Cell Rep 22: 1016-1030, 2018.

This work is licensed under a Creative Commons Attribution-NonCommercial-NoDerivatives 4.0 International (CC BY-NC-ND 4.0) License. 